\title{
Quantum Monte Carlo studies of covalent and metallic clusters: Accuracy of density functional approximations
}

\author{
C. R. Hsing, ${ }^{1}$ C. M. Wei, ${ }^{1,2, *}$ N. D. Drummond, ${ }^{3}$ and R. J. Needs ${ }^{3}$ \\ ${ }^{1}$ Institute of Atomic and Molecular Sciences, Academia Sinica, P.O. Box 23-166, Taipei 10617, Taiwan \\ ${ }^{2}$ Institute of Physics, Academia Sinica, Nankang, Taipei 11529, Taiwan \\ ${ }^{3}$ Theory of Condensed Matter Group, Cavendish Laboratory, University of Cambridge, Cambridge CB3 OHE, United Kingdom
}

(Received 2 April 2008; revised manuscript received 27 January 2009; published 1 June 2009)

\begin{abstract}
To assess the accuracy of exchange-correlation approximations within density functional theory (DFT), diffusion quantum Monte Carlo (DMC) and DFT methods are used to calculate the energies of isomers of three covalently bonded carbon and boron clusters $\left(\mathrm{C}_{20}, \mathrm{~B}_{18}\right.$, and $\left.\mathrm{B}_{20}\right)$, and three metallic aluminum and copper clusters $\left(\mathrm{Al}_{13}, \mathrm{Al}_{55}\right.$, and $\left.\mathrm{Cu}_{13}\right)$. We find that local and semilocal DFT methods predict the same energy ordering as DMC for the metallic clusters but not for the covalent clusters, implying that the DFT functionals are inadequate in such systems. In addition, we find that DFT fails to describe energy reductions arising from Jahn-Teller distortions.
\end{abstract}

DOI: 10.1103/PhysRevB.79.245401

PACS number(s): 61.46.Bc, 02.70.Ss, 36.40.-c, 71.15.Mb

\section{INTRODUCTION}

Aggregates of $\sim 10-10^{6}$ atoms or molecules are known as nanoclusters. They form a bridge between molecules and bulk materials, and are the building blocks of nanoscience. ${ }^{1}$ Furthermore, they often show significant quantum-size effects and irregular changes in properties with increasing cluster size. Clusters have naturally been the subject of numerous experimental and theoretical studies in recent years. ${ }^{2-8}$ There are many potential technological applications of nanoclusters. Several metallic clusters have been shown to be very active catalysts ${ }^{9,10}$ due to their high surface-area-to-volume ratios and unusual surface structures. It may be possible to use nanoclusters in the production of cheap and efficient solar cells. ${ }^{11}$ There has also been much interest in the possibility of using nanoclusters for innovative drug delivery systems. ${ }^{12}$

A crucial first step in studying a cluster is to identify its most stable isomers. The most widely used theoretical method for this purpose is density functional theory (DFT). Unfortunately, DFT predictions of the most stable isomers of covalent clusters sometimes differ from those obtained using more accurate theoretical methods, casting serious doubt on the reliability of DFT results. ${ }^{13-17}$ The accuracy of DFT for metallic clusters has not been tested to the same extent, despite the fact that metallic clusters are of greater technological importance and have been the subject of numerous DFT studies. It is clearly essential to assess the accuracy of DFT over a range of different cluster types. To this end, we have used the highly accurate diffusion quantum Monte Carlo (DMC) method ${ }^{18}$ to generate benchmark energies for both covalent and metallic clusters.

In previous studies, the high-level quantum chemistry coupled-cluster method including single, double, and noniterative perturbative triple excitations $[\operatorname{CCSD}(\mathrm{T})]$ has been used to calculate the energies of three isomers of $\mathrm{C}_{20},{ }^{16,17}$ giving results in reasonable agreement with $\mathrm{DMC}^{13,14}$ but in disagreement with DFT. DMC can be applied to systems ranging from atoms to bulk materials because its computational cost scales as the cube of the system size, which is much more favorable than $\operatorname{CCSD}(\mathrm{T})$, whose cost scales as the seventh power of the system size. This has allowed us to study a wider range of clusters than would have been possible with high-level quantum chemistry methods. The importance of high-quality benchmark calculations lies not only in comparisons with simpler methods such as DFT but also in understanding experimental data. For example, experiments indicate that the most stable isomer of $\mathrm{C}_{20}$ is a ring, ${ }^{19,20}$ whereas the most accurate theoretical studies indicate that the bowl and cage are lower in energy. ${ }^{13,14,16,17}$ The resolution of this paradox is that the experiments are conducted at high temperatures; in fact the ring isomer is calculated to be the most stable when finite-temperature corrections $^{21,22}$ are included. To study the role of finitetemperature effects carefully, both experimental results and highly accurate data for the static-nucleus energy differences between the isomers are required.

In order to examine the accuracy of various DFT exchange-correlation $(\mathrm{XC})$ functionals in predicting the relative energies of cluster isomers and to provide new benchmark data, we have performed DMC calculations for three covalent carbon and boron clusters $\left(\mathrm{C}_{20}, \mathrm{~B}_{18}\right.$, and $\left.\mathrm{B}_{20}\right)$, and three metallic aluminum and copper clusters $\left(\mathrm{Al}_{13}, \mathrm{Al}_{55}\right.$, and $\left.\mathrm{Cu}_{13}\right)$. We compare our DMC results with DFT ones obtained using the local density approximation (LDA), ${ }^{23}$ and the Perdew-Wang 1991 (PW91) ${ }^{24}$ and Perdew-Burke-Ernzerhof $(\mathrm{PBE})^{25}$ generalized gradient approximation (GGA) XC functionals. Our work extends earlier DMC studies of covalent clusters ${ }^{13,14,26,27}$ to a broader range of clusters with strong covalent bonds $(\mathrm{C}$ and $\mathrm{B})$ and metallic bonding ( $\mathrm{Al}$ and $\mathrm{Cu}$ ), the latter including strong electron-correlation effects from the tightly bound $\mathrm{Cu} 3 d$ electrons. From our DMC results, we conclude that LDA and GGA XC functionals are reliable for metallic clusters but not for covalent ones.

Our paper is organized as follows. In Sec. II we briefly describe the computational details of our calculations. In Secs. III A and III B we report the DFT and DMC relative energies of the various cluster isomers that we have studied. In Sec. III C we present an analysis of the correlation between the isomer energies obtained using DMC and DFT. 

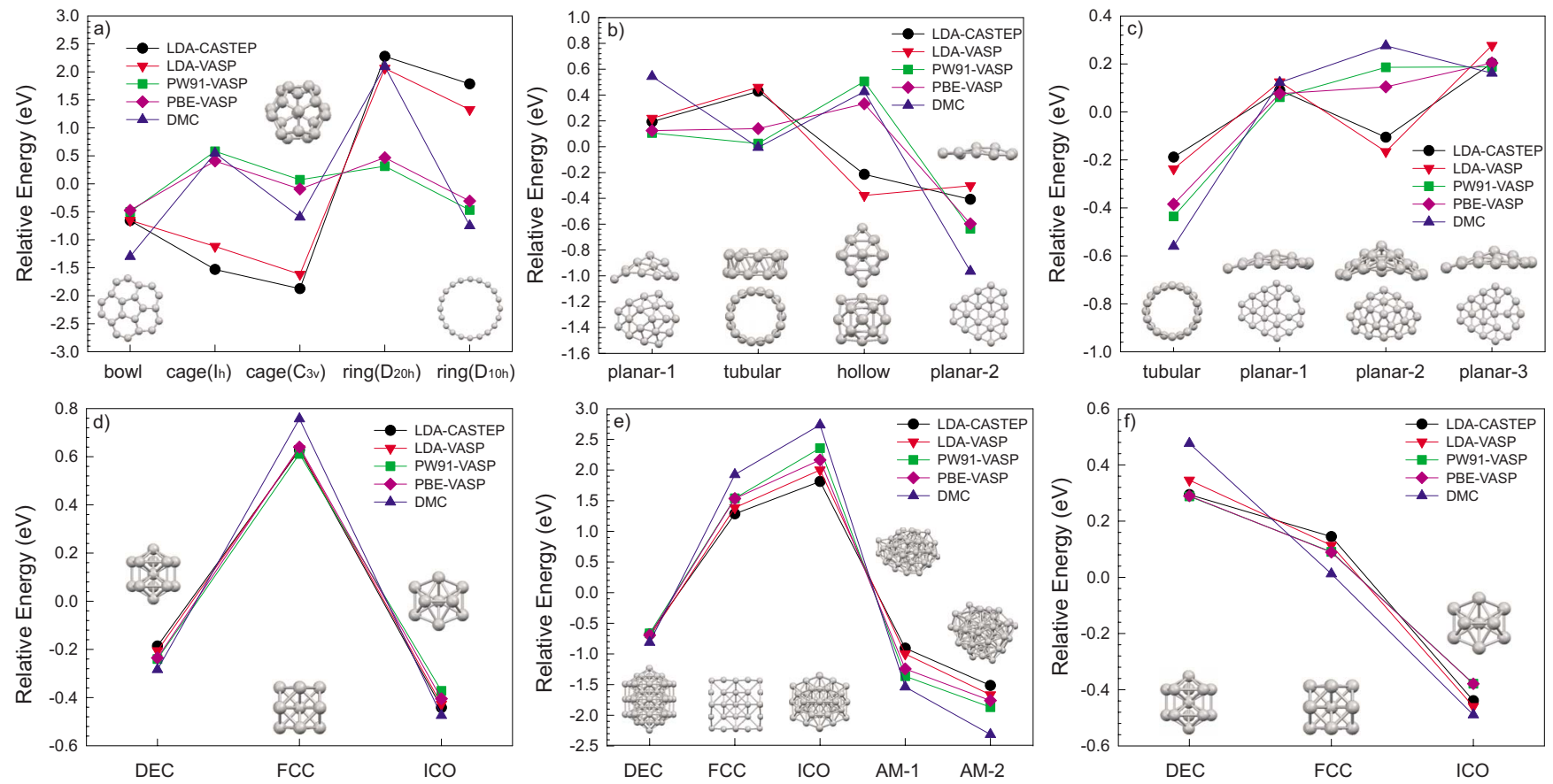

FIG. 1. (Color online) Energy relative to the average for isomers of (a) $\mathrm{C}_{20}$, (b) $\mathrm{B}_{18}$, (c) $\mathrm{B}_{20}$, (d) $\mathrm{Al}_{13}$, (e) $\mathrm{Al}_{55}$, and (f) $\mathrm{Cu}_{13}$ obtained using the DFT-LDA, DFT-PBE, DFT-PW91, and DMC methods. The DMC error bars are about $50 \mathrm{meV}_{\text {for }} \mathrm{C}_{20}$, less than 27 meV for $\mathrm{B}_{18}$ and $\mathrm{B}_{20}$, less than $48 \mathrm{meV}$ for the $\mathrm{Al}$ clusters, and less than $60 \mathrm{meV}$ for $\mathrm{Cu}_{13}$.

Finally, we summarize our findings in Sec. IV.

\section{COMPUTATIONAL DETAILS}

Our DFT calculations were performed with the CASTEP ${ }^{28}$ and VASP ${ }^{29}$ plane-wave DFT codes. In the VASP calculations the electron-ion interaction was described by the projectoraugmented-wave method while in the CASTEP calculations we used norm-conserving Dirac-Fock pseudopotentials ${ }^{30}$ for $\mathrm{C}, \mathrm{B}$, and $\mathrm{Al}$, and an optimized LDA pseudopotential ${ }^{31}$ for $\mathrm{Cu}$. The data presented in Fig. 1 show that the LDA energies obtained using CASTEP and VASP are very similar, demonstrating that the results are insensitive to the choice of pseudopotential. We used plane-wave cutoff energies of at least $1000 \mathrm{eV}$ for the $\mathrm{B}, \mathrm{C}$, and $\mathrm{Cu}$ clusters, and at least 400 $\mathrm{eV}$ for the $\mathrm{Al}$ clusters. The periodic simulation cell had a side length of at least $12 \AA$ in each case in order to eliminate interactions between clusters.

The initial cluster geometries were taken from previous theoretical work. We also performed first-principles molecular dynamics (MD) simulations which enabled us to discover unfound structures. The structures were fully relaxed within DFT-LDA. We used the fixed-node DMC method as implemented in the CASINO code ${ }^{32}$ with the geometries optimized within CASTEP. We used the same pseudopotentials as in the CASTEP calculations because it has been found that DiracFock-based pseudopotentials give superior results to DFT ones in DMC calculations. ${ }^{33}$ Our trial wave functions were of Slater-Jastrow form, where the Slater determinants contained the DFT-LDA orbitals generated by CASTEP. Our Jastrow factors contained electron-electron and electron-ion terms ${ }^{34}$ and the variable parameters were optimized by mini- mizing the variance of the energy. ${ }^{35}$ Electron-electronnucleus Jastrow terms were not found to make a significant improvement to the wave functions. We used a DMC time step of 0.01 a.u. in all our production calculations, apart from those for $\mathrm{Al}_{13}$ and $\mathrm{B}_{18}$, where we used a time step of 0.005 a.u. We verified that the energy differences between isomers were converged with respect to the DMC time step by repeating some of the calculations with half the time step.

For the "G1" set of 55 molecules, Grossman ${ }^{36}$ has shown that the average absolute deviation of the pseudopotential fixed-node DMC atomization energy from the experimental benchmark data is $2.9 \mathrm{kcal} / \mathrm{mol}(0.126 \mathrm{eV})$. We expect the cancellation of fixed-node errors between isomers of the same cluster to be much better than the cancellation that occurs in the calculation of the atomization energy.

\section{RESULTS AND DISCUSSION}

\section{A. Covalent clusters}

We first considered three $\mathrm{C}_{20}$ isomers: a ring of $D_{10 h}$ symmetry, a cage of $C_{3 v}$ symmetry, and a bowl of $C_{5 v}$ symmetry. The isomer energies relative to their average are plotted in Fig. 1(a). The DMC energy of the ring is $0.55 \mathrm{eV}$ higher than that of the bowl while the energy of the cage is $0.15 \mathrm{eV}$ higher still. DFT-PBE and DFT-PW91 calculations give the same energy ordering as DMC while DFT-LDA predicts that the cage has the lowest energy. This suggests that GGAs give a better description of $\mathrm{C}$ clusters than the LDA. Our DMC results are qualitatively consistent with those of previous DMC studies, ${ }^{13,14}$ which used geometries optimized within Hartree-Fock theory, a Gaussian basis set for the orbitals, 
and both pseudopotential and all-electron methods. However, the previous DMC studies found the energy differences between the ring and bowl structures, and cage and ring structures to be 1.1 and $1.0 \mathrm{eV}$, respectively. The likely reason for the discrepancy with our results is the use of Hartree-Fock geometries in the earlier studies. A comparison with coupledcluster results has shown that the energy differences between $\mathrm{C}$ clusters optimized at the Hartree-Fock level are too large. ${ }^{16}$

A $\mathrm{C}_{20}$ cage isomer of $I_{h}$ symmetry may undergo a JahnTeller distortion to a lower-symmetry $C_{3 v}$ structure while a ring structure of $D_{20 h}$ symmetry can undergo a Jahn-Teller distortion to a lower-symmetry $D_{10 h}$ structure. The energy lowering due to these distortions is shown in Fig. 1(a). The $C_{3 v}$ cage is $1.14 \mathrm{eV}$ lower in energy than the $I_{h}$ cage within DMC but within DFT the energy reduction ranges from 0.3 to $0.5 \mathrm{eV}$. For the ring isomer, the DMC energy of the $D_{10 h}$ isomer is $2.84 \mathrm{eV}$ lower than $D_{20 h}$ but the DFT energy reduction ranges from 0.5 to $0.8 \mathrm{eV}$. The discrepancy between the DMC and DFT energy differences demonstrates that DFT with local and semilocal XC functionals underestimates the energy reduction from the Jahn-Teller effect.

We have also studied B clusters. Figures 1(b) and 1(c) show the geometries of different isomers of $\mathrm{B}_{18}$ (a doubleringed tube, a hollow with $O_{h}$ symmetry, and two planar isomers) and $\mathrm{B}_{20}$ (a tube and three planar isomers). The $\mathrm{B}_{20}$ structures were taken from previous calculations ${ }^{15}$ and relaxed at the DFT level, except for the planar-3 isomer [see Fig. 1(c)], which we found using first-principles MD simulations. Our DFT and DMC energies are reported in Figs. 1(b) and 1(c). The DFT-LDA, DFT-PBE, and DMC calculations all predict that the planar-2 isomer of $\mathrm{B}_{18}$ and the double-ring tubular isomer of $\mathrm{B}_{20}$ are the most stable structures.

An earlier first-principles study of $\mathrm{B}_{20}$ also found the tubular isomer to be lowest in energy. ${ }^{15}$ In that work, it was suggested that the planar structure is the most stable for $\mathrm{B}_{n}$ clusters with $n<20$. Our results for $\mathrm{B}_{18}$ support this conjecture. However, the numerical values of the DFT-LDA and DFT-PBE relative energies of the isomers are very different. The DMC results indicate that the PBE functional is more accurate than the LDA. In the LDA expression for the $\mathrm{XC}$ hole, the density prefactor is the local density instead of the nonlocal density that appears in the exact XC hole formula. This causes the LDA to favor compact structures. In their study of $\mathrm{C}_{20}$, Grossman et al. ${ }^{13}$ found that the LDA favors the more compact cage structure over the ring or bowl isomers. In $\mathrm{B}_{18}$, the planar- 1 and hollow geometries are more compact than the tubular structure while in $\mathrm{B}_{20}$ the planar-2 structure is more compact than the planar-1 and planar-3 structures; the results in Figs. 1(b) and 1(c) show that the LDA again favors the more compact structures.

\section{B. Metallic clusters}

We have also performed DFT and DMC calculations for $\mathrm{Al}$ and $\mathrm{Cu}$ clusters. We investigated three high-symmetry isomers of the magic-number clusters $\mathrm{Al}_{13}, \mathrm{Al}_{55}$, and $\mathrm{Cu}_{13}$; the icosahedral (ICO), decahedral (DEC), and cuboctahedral
(FCC) structures. To perform a more rigorous comparison between DMC and DFT, we also examined two amorphous structures of $\mathrm{Al}_{55}$ (AM-1 and AM-2), which we found by first-principles MD simulation. The amorphous isomers have lower energies than the high-symmetry structures. The relative energies of the Al clusters are shown in Figs. 1(d) and 1(e) and those of $\mathrm{Cu}_{13}$ are shown in Fig. 1(f). The LDA performs relatively well in metallic clusters compared with covalent clusters because the valence-electron distribution is comparatively smooth. Indeed, we find that the DFT-LDA, DFT-GGA, and DMC energy orderings are in agreement. However, the GGAs give slightly more accurate energy differences than the LDA, especially in $\mathrm{Al}_{55}$ isomers.

\section{Correlation analysis}

The extent to which DFT is a reliable predictor of the relative stability of the isomers can be further quantified by examining the correlation between the DFT and DMC energies. For each cluster we define an $n$-dimensional displacement vector $\overrightarrow{\mathfrak{D}}=\left(r_{1}, r_{2}, \ldots, r_{n}\right)$, where $n$ is the number of isomers of the cluster, $r_{i}=E_{i}-\bar{E}$ for each isomer $i$, and $\bar{E}$ is the average energy of the isomers. The correlation of the DFT and DMC energy data is given by

$$
\operatorname{corr}\left(E^{\mathrm{DFT}}, E^{\mathrm{DMC}}\right) \equiv \cos (\theta)=\frac{\overrightarrow{\mathfrak{D}}^{\mathrm{DFT}} \cdot \overrightarrow{\mathfrak{D}}^{\mathrm{DMC}}}{\left(\left|\overrightarrow{\mathfrak{D}}^{\mathrm{DFT}} \| \overrightarrow{\mathfrak{D}}^{\mathrm{DMC}}\right|\right)},
$$

while the relative amplitude,

$$
L=\frac{\left|\overrightarrow{\mathfrak{D}}^{\mathrm{DFT}}\right|}{\left|\overrightarrow{\mathfrak{D}}^{\mathrm{DMC}}\right|},
$$

quantifies the extent to which DFT tends to overestimate or underestimate energy differences. The DFT and DMC energies are identical up to a constant shift if and only if $\cos (\theta)=1$ and $L=1$.

Numerical results for $\cos (\theta)$ and $L$ for the different clusters and $\mathrm{XC}$ functionals are given in Table I. These results make the conclusions given in the preceding paragraphs strikingly clear: $(1) \cos (\theta)$ is close to unity for metallic clusters but is significantly lower for covalent clusters. DFT is therefore much better at predicting the energy ordering of metallic clusters than covalent ones. It can also be seen that the value of $\cos (\theta)$ for a cluster of the transition metal $\mathrm{Cu}$ is intermediate between the values for the covalent and simple metallic clusters. (2) The different XC functionals predict the relative stabilities of metallic clusters consistently but this is not the case for the covalent clusters. The value of $\cos (\theta)$ for metallic clusters ranges from 1 to 0.976 and $L$ ranges from 0.708 to 0.858 . However, for covalent clusters, $\cos (\theta)$ ranges from 0.989 to 0.297 and $L$ ranges from 0.312 to 1.189 . (3) The GGA functionals are only slightly better than the LDA for metallic clusters although they are significantly better than the LDA for covalent clusters. Another interesting finding is that $L<1$ for all the metallic and most of the covalent clusters studied, showing that DFT tends to predict smaller energy differences than DMC. 
TABLE I. The correlation $\cos (\theta)$ and relative amplitude $L$ of the two displacement vectors $\overrightarrow{\mathfrak{D}}^{\mathrm{DFT}}$ and $\overrightarrow{\mathfrak{D}}^{\mathrm{DMC}}$ for different clusters. The DFT energies were calculated using VASP.

\begin{tabular}{|c|c|c|c|c|c|c|}
\hline \multirow[b]{2}{*}{ Cluster } & \multicolumn{3}{|c|}{$\cos (\theta)$} & \multicolumn{3}{|c|}{$L$} \\
\hline & LDA & PW91 & PBE & LDA & PW91 & PBE \\
\hline $\mathrm{C}_{20}$ & 0.523 & 0.750 & 0.923 & 1.189 & 0.353 & 0.312 \\
\hline $\mathrm{B}_{18}$ & 0.297 & 0.911 & 0.933 & 0.592 & 0.690 & 0.596 \\
\hline $\mathrm{B}_{20}$ & 0.535 & 0.989 & 0.961 & 0.639 & 0.781 & 0.691 \\
\hline $\mathrm{Al}_{13}$ & 0.998 & 1.000 & 1.000 & 0.844 & 0.805 & 0.845 \\
\hline $\mathrm{Al}_{55}$ & 0.998 & 0.999 & 1.000 & 0.722 & 0.837 & 0.788 \\
\hline $\mathrm{Cu}_{13}$ & 0.976 & 0.978 & 0.979 & 0.858 & 0.708 & 0.710 \\
\hline
\end{tabular}

\section{SUMMARY}

In summary, we have used DMC methods to calculate the relative energies of isomers of six different nanoclusters. For $\mathrm{C}$ and $\mathrm{B}$ clusters, different approximate DFT XC functionals yield different energy orderings and the relative DFT energies sometimes differ significantly from the DMC results. GGA functionals are more accurate than the LDA in these systems. DFT fails to give an adequate description of energy differences caused by the Jahn-Teller effect in $\mathrm{C}_{20}$ isomers. Our results strongly suggest that, in studies of covalently bonded clusters, DFT calculations using local (LDA) or semilocal (PW91 or PBE) XC functionals should only be used with extreme caution. On the other hand, DFT-LDA, DFT-GGA, and DMC calculations give accurate energy differences for isomers of $\mathrm{Al}$ and $\mathrm{Cu}$ clusters. The DFT ap- proach therefore appears to be rigorous and reliable in studies of metallic clusters.

\section{ACKNOWLEDGMENTS}

This work was supported in part by the National Science Council of Taiwan under Grants No. 96-2628-M001-006MY3 (C.R.H. and C.M.W.) and No. 96-2120-M-002-010 (C.M.W.). We also acknowledge the Academia Sinica Research Program on Nanoscience and Nanotechnology, the National Center for Theoretical Sciences (NCTS) and computing resources from the National Center for HighPerformance Computing (NCHC) in Taiwan (C.R.H. and C.M.W.). N.D.D. acknowledges support from Jesus College, Cambridge. *cmw@phys.sinica.edu.tw

${ }^{1}$ F. Baletto and R. Ferrando, Rev. Mod. Phys. 77, 371 (2005).

${ }^{2}$ E. Roduner, Chem. Soc. Rev. 35, 583 (2006).

${ }^{3}$ L. Belau, S. E. Wheeler, B. W. Ticknor, M. Ahmed, S. R. Leone, W. D. Allen, H. F. Schaefer III, and M. A. Duncan, J. Am. Chem. Soc. 129, 10229 (2007).

${ }^{4}$ E. Wahlstrom, N. Lopez, R. Schaub, P. Thostrup, A. Ronnau, C. Africh, E. Laegsgaard, J. K. Nørskov, and F. Besenbacher, Phys. Rev. Lett. 90, 026101 (2003).

${ }^{5}$ G. Rossi, A. Rapallo, C. Mottet, A. Fortunelli, F. Baletto, and R. Ferrando, Phys. Rev. Lett. 93, 105503 (2004).

${ }^{6}$ J. Li, X. Li, H. J. Zhai, and L. S. Wang, Science 299, 864 (2003).

${ }^{7}$ X. Li, A. Grubisic, S. T. Stokes, J. Cordes, G. F. Gantefor, K. H. Bowen, B. Kiran, M. Willis, P. Jena, R. Burgert, and H. Schnockel, Science 315, 356 (2007).

${ }^{8}$ P. Gruene, D. M. Rayner, B. Redlich, A. F. G. van der Meer, J. T. Lyon, G. Meijer, and A. Fielicke, Science 321, 674 (2008).

${ }^{9}$ C. R. Henry, Surf. Sci. Rep. 31, 231 (1998).

${ }^{10}$ J. D. Aiken III and R. G. Finke, J. Mol. Catal. A: Chem. 145, 1 (1999).

${ }^{11}$ S. Pillai, K. R. Catchpole, T. Trupke, and M. A. Green, J. Appl. Phys. 101, 093105 (2007).

${ }^{12}$ C. Sun, J. S. H. Lee, and M. Q. Zhang, Adv. Drug Delivery Rev. 60, 1252 (2008).
${ }^{13}$ J. C. Grossman, L. Mitas, and K. Raghavachari, Phys. Rev. Lett. 75, 3870 (1995).

${ }^{14}$ S. Sokolova, A. Lüchow, and J. B. Anderson, Chem. Phys. Lett. 323, 229 (2000)

${ }^{15}$ W. An, S. Bulusu, Y. Gao, and X. C. Zeng, J. Chem. Phys. 124, 154310 (2006).

${ }^{16}$ S. Grimme and C. Mück-Lichtenfeld, ChemPhysChem 3, 207 (2002).

${ }^{17}$ W. An, Y. Gao, S. Bulusu, and X. C. Zeng, J. Chem. Phys. 122, 204109 (2005).

${ }^{18}$ W. M. C. Foulkes, L. Mitas, R. J. Needs, and G. Rajagopal, Rev. Mod. Phys. 73, 33 (2001).

${ }^{19}$ P. Dugourd, R. R. Hudgins, J. M. Tenenbaum, and M. F. Jarrold, Phys. Rev. Lett. 80, 4197 (1998).

${ }^{20}$ H. Prinzbach, A. Weiler, P. Landenberger, F. Wahl, J. Wörth, L. T. Scott, M. Gelmont, D. Olevano, and B. von Issendorff, Nature (London) 407, 60 (2000)

${ }^{21}$ C. J. Brabec, E. B. Anderson, B. N. Davidson, S. A. Kajihara, Q.-M. Zhang, J. Bernholc, and D. Tomanek, Phys. Rev. B 46, 7326 (1992).

${ }^{22}$ A. Castro, M. A. L. Marques, J. A. Alonso, G. F. Bertsch, K. Yabana, and A. Rubio, J. Chem. Phys. 116, 1930 (2002).

${ }^{23}$ J. P. Perdew and A. Zunger, Phys. Rev. B 23, 5048 (1981).

${ }^{24}$ J. P. Perdew, J. A. Chevary, S. H. Vosko, K. A. Jackson, M. R. Pederson, D. J. Singh, and C. Fiolhais, Phys. Rev. B 46, 6671 
(1992).

${ }^{25}$ J. P. Perdew, K. Burke, and M. Ernzerhof, Phys. Rev. Lett. 77, 3865 (1996)

${ }^{26}$ P. R. C. Kent, M. D. Towler, R. J. Needs, and G. Rajagopal, Phys. Rev. B 62, 15394 (2000).

${ }^{27}$ L. Mitas, J. C. Grossman, I. Stich, and J. Tobik, Phys. Rev. Lett. 84, 1479 (2000).

${ }^{28}$ M. D. Segall, P. J. D. Lindan, M. J. Probert, C. J. Pickard, P. J. Hasnip, S. J. Clark, and M. C. Payne, J. Phys.: Condens. Matter 14, 2717 (2002).

${ }^{29}$ G. Kresse and J. Hafner, Phys. Rev. B 49, 14251 (1994); G. Kresse and J. Furthmüller, Comput. Mater. Sci. 6, 15 (1996).

${ }^{30}$ J. R. Trail and R. J. Needs, J. Chem. Phys. 122, 014112 (2005); 122, 174109 (2005).
${ }^{31}$ A. M. Rappe, K. M. Rabe, E. Kaxiras, and J. D. Joannopoulos, Phys. Rev. B 41, 1227 (1990).

${ }^{32}$ R. J. Needs, M. D. Towler, N. D. Drummond, and P. López Ríos, CASINO Version 2.1 User Manual (Cambridge University Press, Cambridge, 2007)

${ }^{33}$ C. W. Greeff and W. A. Lester, Jr., J. Chem. Phys. 109, 1607 (1998).

${ }^{34}$ N. D. Drummond, M. D. Towler, and R. J. Needs, Phys. Rev. B 70, 235119 (2004).

${ }^{35}$ C. J. Umrigar, K. G. Wilson, and J. W. Wilkins, Phys. Rev. Lett. 60, 1719 (1988); N. D. Drummond and R. J. Needs, Phys. Rev. B 72, 085124 (2005).

${ }^{36}$ J. C. Grossman, J. Chem. Phys. 117, 1434 (2002). 